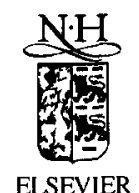

ELSEVIER

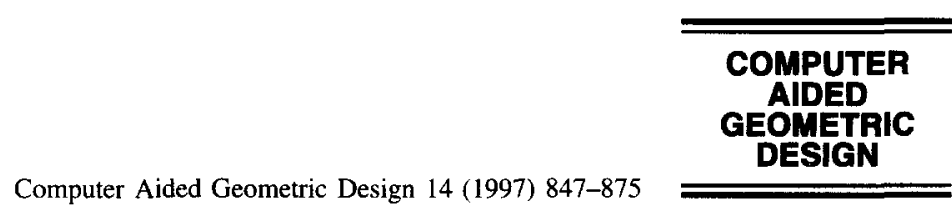

\title{
Bivariate Hermite subdivision
}

\author{
Ruud van Damme ${ }^{1}$ \\ University of Twente, Department of Mathematics, P.O. Box 217, 7500 AE Enschede, The Netherlands
}

Received June 1996; revised January 1997

\begin{abstract}
A subdivision scheme for constructing smooth surfaces interpolating scattered data in $\mathbb{R}^{3}$ is proposed. It is also possible to impose derivative constraints in these points. In the case of functional data, i.e., data are given in a properly triangulated set of points $\left\{\left(x_{i}, y_{i}\right)\right\}_{i=1}^{N}$ from which none of the pairs $\left(x_{i}, y_{i}\right)$ and $\left(x_{j}, y_{j}\right)$ with $i \neq j$ coincide, it is proved that the resulting surface (function) is $C^{1}$. The method is based on the construction of a sequence of continuous splines of degree 3 . Another subdivision method, based on constructing a sequence of splines of degree 5 which are once differentiable, yields a function which is $C^{2}$ if the data are not 'too irregular'. Finally the approximation properties of the methods are investigated. (c) 1997 Elsevier Science B.V.
\end{abstract}

Keywords: Hermite interpolation; Subdivision; Bivariate splines

AMS classification: 41A15; 65D05; 65D07

\section{Introduction}

Once given a triangulated data set several existing surface generating schemes are based on a kind of subdivision rule applied to this initial triangulation. Mostly, the process of repeated subdivision closely resembles a splitting technique, and is therefore local in the sense that each triangle is subdivided separately. Each triangle in the parameter domain is subdivided in such a way that the new frame of edges constitutes a finer triangulation like the Clough-Tocher and Powell-Sabin splits. In the parametric curve case, at each step, the corners of a polygon are cut such that the sequence of polygons converges to a smooth limiting curve, see e.g., (de Boor, 1987). Similar algorithms have been formulated for subdivision of an arbitrary network of closed polygons, see the schemes described in (Catmull and Clark, 1987; Doo and Sabin, 1978); these

15-mail: vandamme@math.utwente.nl.

0167-8396/97/\$17.00 Copyright (C) 1997 Published by Elsevier Science B.V. All rights reserved. PII S0167-8396(97)00009-5 
schemes are not interpolatory. A 'butterfly' scheme that yields $C^{1}$ interpolating surfaces (except possibly in the original vertices of the dataset) is described in (Dyn et al., 1990). Also an interpolating and convexity preserving subdivision scheme exists (Dyn et al., 1992), but it is rather complicated in its implementation. For a survey of subdivision algorithms for curve and surface design, see (Cavaretta and Micchelli, 1989; Cavaretta et al., 1991).

The main difference of the method described here with most existing subdivision schemes, is that besides positional information also derivative information is taken into account (see (Merrien, 1994) for a similar method, however). If this extra information is not available one can of course estimate it. That is, from a $3 \mathrm{D}$ triangulation with normals attached to each vertex a finer triangulation is constructed, with vertices and normals through which the final surface will pass. The gain in taking derivative data into account is that one can more intuitively define suitable subdivision rules. A drawback is that the proposed rules are nonlinear in the data and this makes it very hard to analyse them theoretically. In the case of functional data, this analysis can be performed, however.

In Section 2 the principle of the subdivision scheme, applied to parametric surfaces is described. The main idea is to construct a sequence of parametric surfaces which belong to a spline space of certain degree $d$ and smoothness $\rho$, denoted by $S_{d}^{\rho}$. The triangulation is then refined every step. The space $S_{2}^{0}$ turns out to be too small: the data must be convex, and it is not clear whether the resulting surface is smooth even in the case that the data are strictly convex. The space $S_{3}^{0}$ as a starting point seems very well suited.

In Section 3 the subdivision scheme for functional data is stated. In that case it is proved that the resulting surface is of class $C^{1}$ (Section 4). Next it is shown that there exists a method that even yields a $C^{2}$ surface if one takes the spline space $S_{5}^{1}$ as a starting point, provided the data are not 'too scattered'. This is explained in Section 5. In the final section the approximation properties of the methods are examined.

\section{Hermite subdivision for parametric surfaces}

In this section the principle of the subdivision scheme is described. The main idea is to construct a sequence of surfaces which belong to a spline space from $S_{d}^{\rho}$. We investigate methods which use $S_{2}^{0}, S_{3}^{0}$ and $S_{5}^{1}$. For short we refer to the 'quadratic method' if splines of degree two are used, etc.

The triangulation is refined every step, and in this paper we restrict ourselves to Powell-Sabin splits, i.e., every edge is split in two, and every triangle into four parts (see Fig. 1).

Although it is possible to use the space $S_{2}^{0}$ as a starting point if the data set is convex, the space $S_{3}^{0}$ seems to be a better choice: as will be shown in the sequel in the case of functional data it can actually be proved that the resulting surface is of class $C^{1}$.

First consider data in $\mathbb{R}^{2}$ through which a smooth curve has to be constructed. The method described here is not really new but it serves as an example, which is later generalised to the construction of surfaces in three dimensions. 

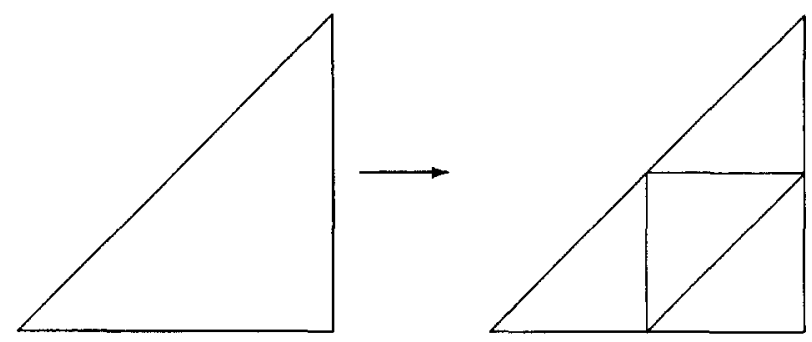

Fig. 1. The Powell-Sabin split.

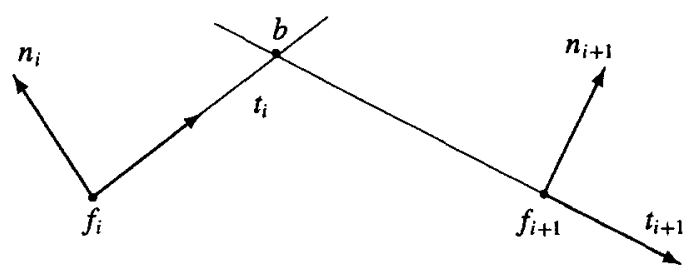

Fig. 2. Construction of a quadratic curve.

\subsection{Curves}

Consider data $f_{i} \in \mathbb{R}^{2}, i=0, \ldots, N$. As input are given two data points with corresponding normals, and define an intermediate point and normal as convex combinations. If the normals are not supplied, one can of course use estimates of these normals. This approach results in the following quadratic curve algorithm that works for convex data. Given are two points $f_{i}, f_{i+1} \in \mathbb{R}^{2}$ with corresponding (compatible) normals $n_{i}$, $n_{i+1} \in \mathbb{R}^{2}$, see Fig. 2. Now compute $b \in \mathbb{R}^{2}$ as the intersection point of the tangent lines through $f_{i}$ and $f_{i+1}$ with unit tangent vectors $t_{i}, t_{i+1} \in \mathbb{R}^{2}$, perpendicular to the respective normals and then set the new data by

$$
f_{i+1 / 2}=\frac{1}{4}\left(f_{i}+2 b+f_{i+1}\right), \quad t_{i+1 / 2}=\frac{f_{i+1}-f_{i}}{\left\|f_{i+1}-f_{i}\right\|},
$$

and the normal $n_{i+1 / 2}$ perpendicular to $t_{i+1 / 2}$. Clearly, this subdivision process converges to a quadratic parametric curve, since it is equivalent to the de Casteljau algorithm for generating the point with parameter value $\tau=1 / 2$.

Now, we describe a similar cubic algorithm which can handle more arbitrary positional and normal data in $\mathbb{R}^{2}$. Again assume that are given two points $f_{i}, f_{i+1} \in \mathbb{R}^{2}$ with unit normals $n_{i}, n_{i+1} \in \mathbb{R}^{2}$, see Fig. 3 . We want to choose an intermediate point $f_{i+1 / 2}$ with corresponding normal $n_{i+1 / 2}$. Led by the form of a cubic Bernstein-Bézier curve we adopt the following strategy, see (Cottin and van Damme, 1994). Let $L \subset \mathbb{R}^{2}$ be a line with direction $n_{i+1}-n_{i}$, i.e., perpendicular to the average of the given normals $n_{i}$ and $n_{i+1}$. Then construct control points $b_{1}, b_{2} \in \mathbb{R}^{2}$ as in the functional case by first projecting $f_{i}$ and $f_{i+1}$ onto the line $L$ to obtain Bézier sites $x_{0}, x_{3} \in \mathbb{R}^{2}$, defining $x_{1}$, $x_{2} \in \mathbb{R}^{2}$, so that $\left\|x_{0}-x_{1}\right\|=\left\|x_{1}-x_{2}\right\|=\left\|x_{2}-x_{3}\right\|$. Next take $b_{1}$ as the intersection point of the line through $f_{i}$ with direction the tangent vector $t_{i}$ (which is perpendicular 


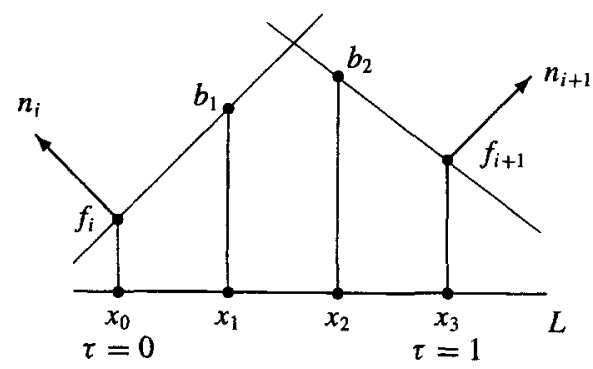

Fig. 3. Construction of a cubic curve.
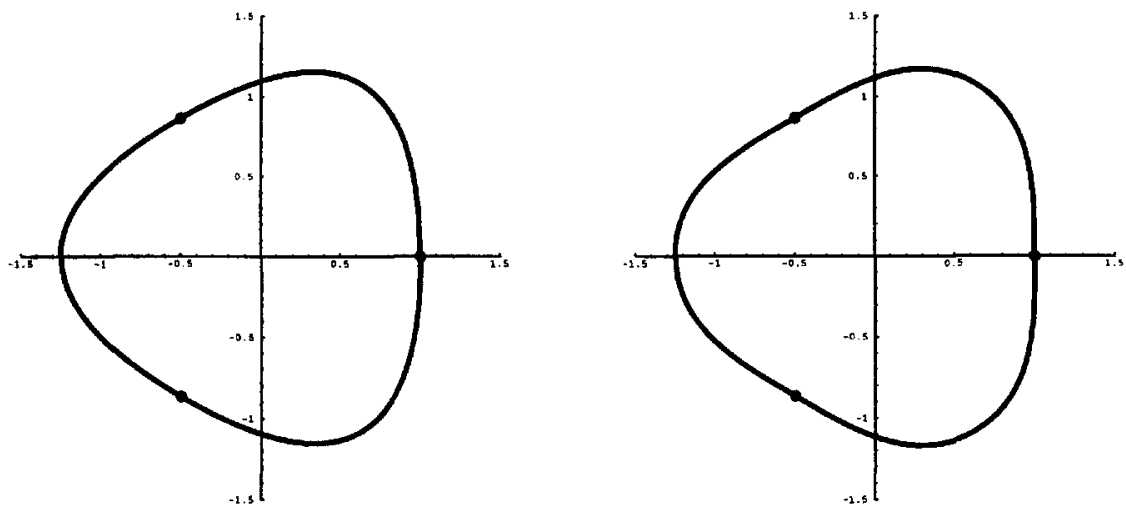

Fig. 4. Quadratic and cubic algorithm to three points from the unit circle.
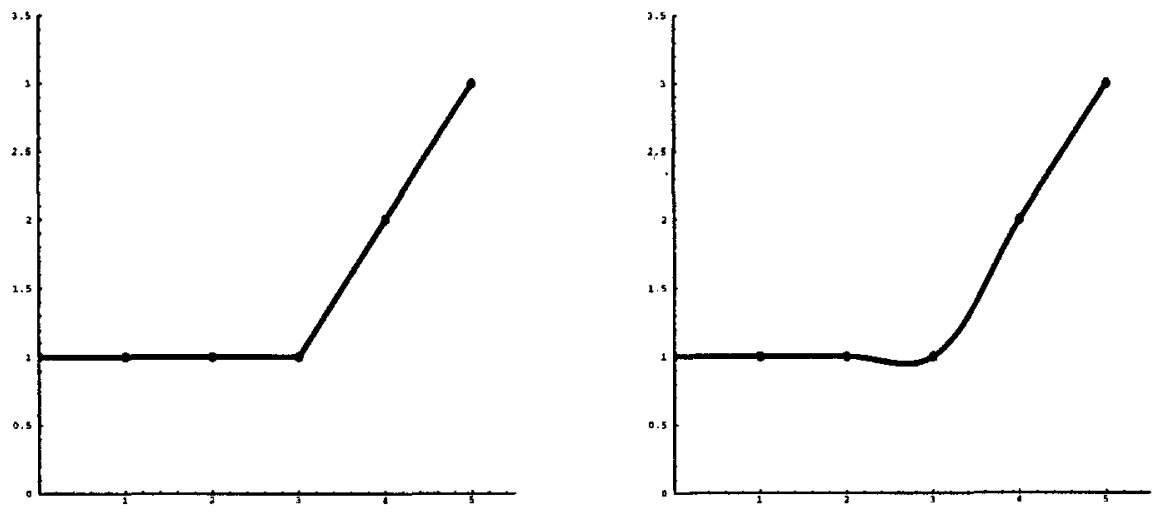

Fig. 5. Quadratic and cubic algorithm to data from a kinked line: $f(x)=1+\max (0, x-3)$.

to normal $n_{i}$ ) and the line through $x_{1}$ with direction $n_{i}+n_{i+1} ; b_{2}$ is found analogously. Similar to (2.1) the new data are derived from the cubic Bézier curve at $\tau=1 / 2$, hence

$$
f_{i+1 / 2}=\frac{1}{8}\left(f_{i}+3 b_{1}+3 b_{2}+f_{i+1}\right), \quad t_{i+1 / 2}=\frac{f_{i+1}+b_{2}-b_{1}-f_{i}}{\left\|f_{i+1}+b_{2}-b_{1}-f_{i}\right\|},
$$


and the normal $n_{i+1 / 2}$ perpendicular to $t_{i+1 / 2}$. (This method also works in degenerate cases, e.g., if $f_{i+1}+b_{2}-b_{1}-f_{i}=0$, see (Cottin and van Damme, 1994).) Repeating this rule converges to a curve that passes through the initial data; the limiting curve will not be a cubic curve in general.

In Figs. 4 and 5 results are shown of some experiments that were carried out for both the quadratic and the cubic scheme. In both cases the data are chosen convex, so that the quadratic as well as the cubic algorithm could be employed. Observe that the quadratic scheme is convexity preserving (and hence not necessarily smooth!), whereas the cubic algorithm yields smooth curves, which need not be convex.

\subsection{Surfaces}

What about a generalisation to surfaces in $\mathbb{R}^{3}$ ? A $3 \mathrm{D}$ triangulation $\mathcal{T}$ defines a continuous surface through the data points. Starting from a given triangulation with normals attached to the vertices, we shall define intermediate points, where we employ the curve algorithms. The old vertices and the new vertices are connected by edges so that a finer triangulation is obtained. Adding normals to the new vertices then completes the subdivision procedure. Here is a short description of the algorithm:

(1) Assigning data to old vertices:

Positional values and normals at the old vertices remain unchanged.

(2) Fill in old patches:

In each old triangle $T$ with vertices $x_{0}, x_{1}, x_{2}$, we fit a quadratic/cubic patch, interpolating the vertices and corresponding normals. To this end, first the boundary curves in one of the two ways as described above is defined, see Figs. 2 and 3. This does not work directly: before this can be done, a plane has to be fixed, in which a boundary curve should lie. (Of course there is no need that they should be in a plane, but this is the simplest choice.) For two vertices $x_{1}, x_{2}$ of $T$, we have chosen this plane such that it contains the edge $\left(x_{1}, x_{2}\right)$ and the direction $n$, which is the mean of the normal vectors at the two vertices.

Now consider Fig. 6. In the quadratic case all Bézier points (the black dots) are now determined. Notice that for the cubic case the points $m_{0}$ and $m_{3}$ are not yet fixed by the local data $x_{0}, \ldots, x_{3}, n_{0}, \ldots, n_{3}$, hence they have to be assigned. A sensible local method is to take $m_{0}$ as a symmetric affine combination of the surrounding control points, i.e.,

$$
\begin{aligned}
m_{0}= & \frac{1}{3}\left(\alpha\left(b_{0,1}+b_{1,0}+b_{0,2}+b_{2,0}+b_{1,2}+b_{2,1}\right)\right. \\
& \left.+(1-2 \alpha)\left(x_{0}+x_{1}+x_{2}\right)\right)
\end{aligned}
$$

where $\alpha$ is a real-valued parameter.

(3) Assigning data to new vertices:

From each of the boundary curves an intermediate point is obtained by computing the curve halfway, i.e., by setting it as in (2.1), respectively (2.2).

The normal at the intermediate point between $x_{1}$ and $x_{2}$ is chosen as the mean of the normals in this point from the two adjacent patches. 

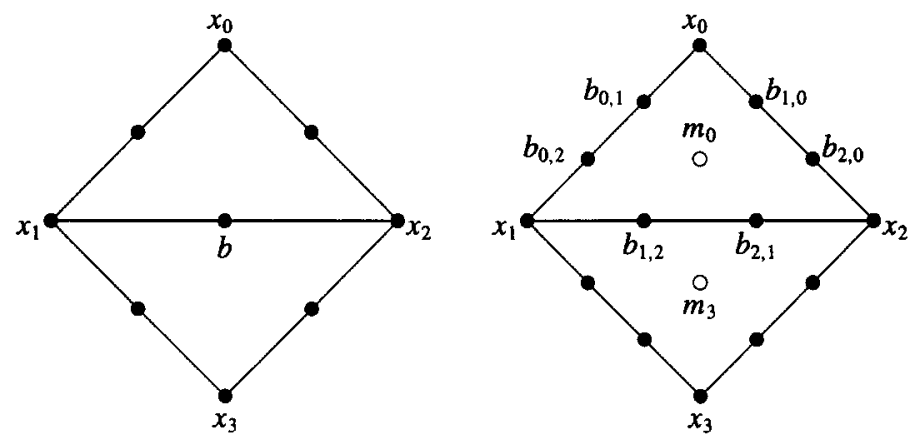

Fig. 6. Quadratic and cubic subdivision.
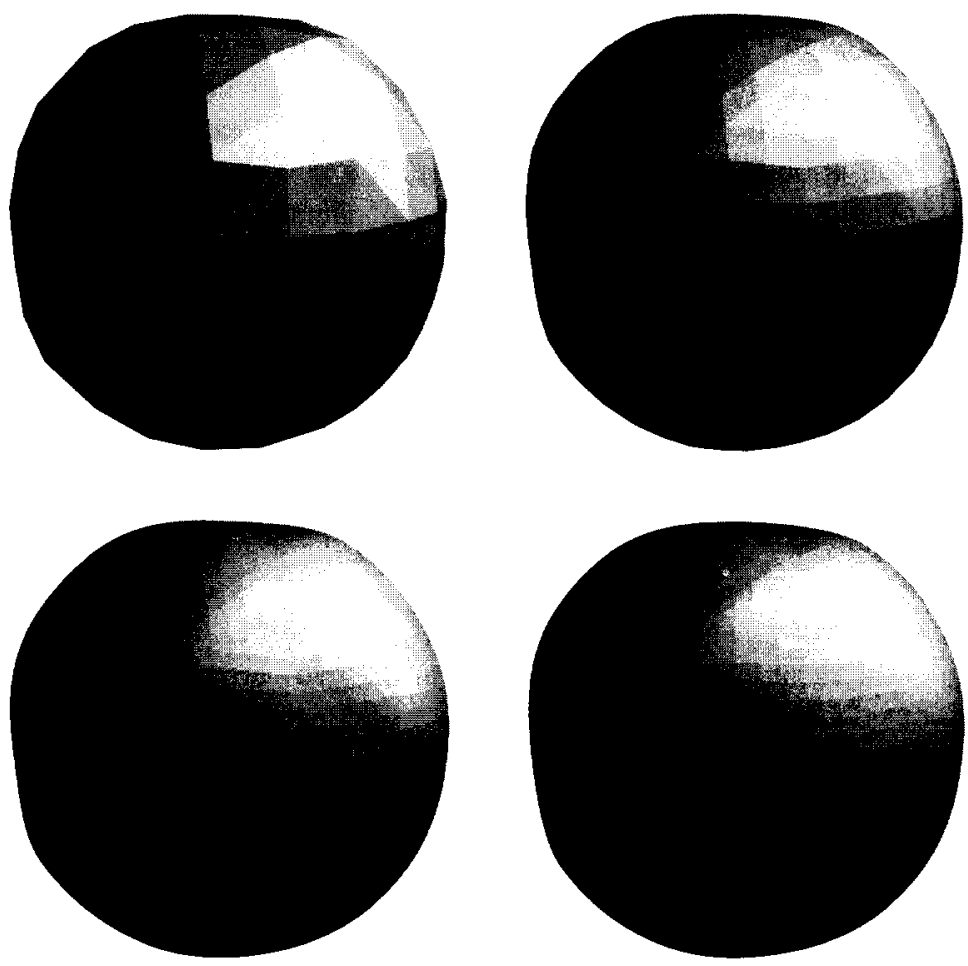

Fig. 7. 3, 4 and 5 cubic and 5 quadratic subdivision steps for data on a regular octahedron.

Fig. 7 shows three subsequent steps for a triangulation of a regular octahedron for the cubic algorithm. The last picture shows the result of five iterations of the quadratic algorithm. A glance at the picture suggests that this method is not convexity preserving: one can actually prove that the quadratic method (in contrast with the curve case) does not preserve convexity in general. In Fig. 8 the final result for the cubic case is depicted after six subdivision steps. 


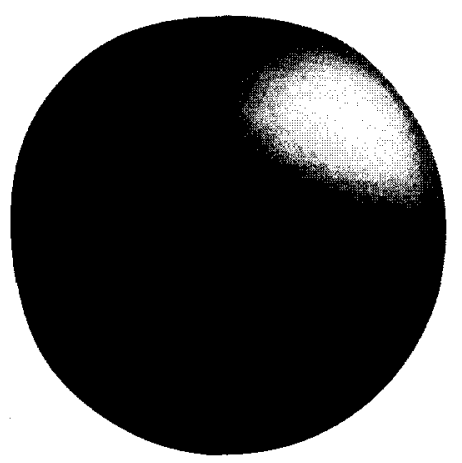

Fig. 8. Limiting cubic surface for subdivision steps for data on a regular octahedron.

All pictures suggest that the algorithm meets our demand of yielding smooth interpolating surfaces. It is not clear how to investigate the smoothness of the resulting surface in the case of parametric nonlinear schemes.

In the next section we restrict ourselves to subdivision of data drawn from a function. The subdivision of the two spatial coordinates is done with a regular Powell-Sabin split, and for the subdivision scheme for the function-values an analogon is made with the method described in this section. (The term 'regular' stands for the fact that every edge is exactly halved.)

\section{Hermite subdivision for bivariate data}

In this section a subdivision scheme for functional data is presented, i.e., it is assumed data are given (a function value and two derivatives) in points $\left\{\left(x_{i}, y_{i}\right)\right\}_{i=1}^{N}$ from which none of the pairs $\left(x_{i}, y_{i}\right)$ and $\left(x_{j}, y_{j}\right)$ with $i \neq j$ coincide. For subdivision in the spatial coordinates $x$ and $y$ a regular Powell-Sabin split is chosen as depicted in Fig. 1.

Here the cubic method is described, whereas in Section 5 the quintic method is discussed. In contrast with the parametric case there is now no freedom to choose the planes in which the boundary curves lie. These curves are now completely fixed by the function values and first derivative data in the vertices of the triangulation $\mathcal{T}$. Together with the function values and derivatives the total Bézier net in every triangle of $\mathcal{T}$ is then fixed, using (2.3).

Remark 3.1. A related subdivision scheme was discussed in (Merrien, 1994). There no connection was made with splines, and there the proof of smoothness seems more complicated.

The explicit form of the subdivision scheme is given later, here we give a description how it works. Given values of the function and both derivatives at some level, we proceed as follows:

(1) Assigning data to old vertices:

Function values and derivatives at the old vertices remain unchanged. 

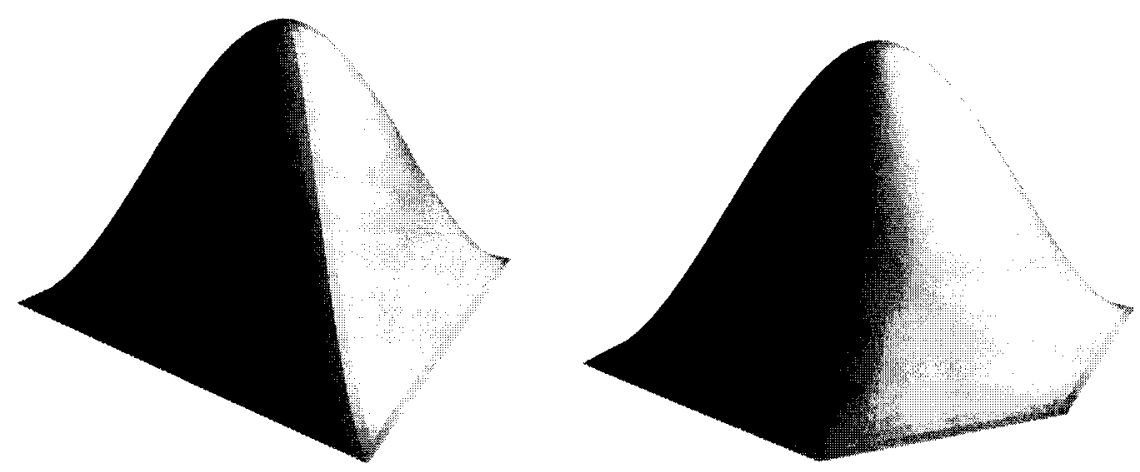

Fig. 9. 5 cubic subdivision steps to cardinal 4 and cardinal 5 .

(2) Fill in old patches:

In each old triangle $T$ with vertices $x_{0}, x_{1}, x_{2}$ we fit a cubic patch, interpolating the old vertices and its derivatives. The remaining Bézier point is fixed according to $(2.3)$.

(3) Assigning data to new vertices:

As the edges are subdivided in two equal parts (a regular Powell-Sabin split) a new vertex lies in the middle of an old edge, i.e., at the boundary of two old triangles. Both these triangles have a Bézier net, describing a $C^{0}$ function. The function value of such a new vertex equals the value of this function halfway the edge, as well as the derivative in the direction of the edge. The derivative perpendicular to the edge is assigned as the average of the two derivatives perpendicular to the edge at the same point.

Fig. 9 shows the result after the fifth step: a vertex $x_{0} \in \mathbb{R}^{2}$ is surrounded by four respectively five randomly chosen other vertices $x_{j} \in \mathbb{R}^{2}$ and the data are given by

$$
f\left(x_{j}\right)=\delta_{j, 0}, \quad f_{x}\left(x_{j}\right)=f_{y}\left(x_{j}\right)=0, \quad j=0, \ldots, 4, \text { respectively } 5 .
$$

Here $f_{x}\left(f_{y}\right)$ stands for the partial derivative of $f$ with respect to $x(y)$.

The approximation of the standard Franke test function (with exact first derivatives) on the standard 36 data set (Franke, 1982) is shown in Fig. $10(\alpha=3 / 4)$.

\section{Proof of convergence for the functional case}

In this section it is proved that the cubic method described in the previous section indeed gives rise to a $C^{1}$ surface for a range of $\alpha$-parameters. In the first subsection the analysis is performed on a type I triangulation. Possibly the analysis can be done using the techniques developed in (Cavaretta et al., 1991; Dyn and Levin, 1995)--here a slightly different approach is followed, as the same strategy is needed later on, when interpolation of scattered data is discussed.

A range of $\alpha$ can be given for which the subdivision scheme converges, and a range for which $C^{1}$ is guaranteed. In the next subsection $C^{1}$ convergence is proved in the case 


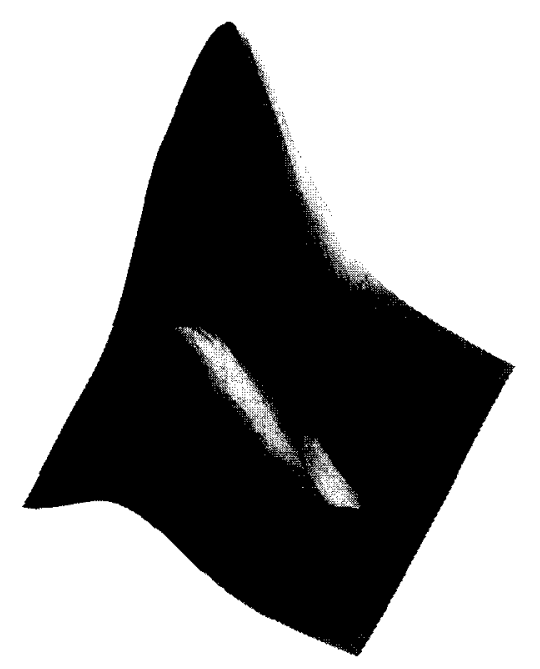

Fig. 10. Cubic subdivision to Franke's function.

of scattered data for one specific $\alpha$-value, namely $\alpha=3 / 4$. For this value the complexity of the proof reduces considerably. The choice $\alpha=3 / 4$ is also special for another reason: in that case the subdivision scheme reproduces quadratic polynomials, i.e., if data are taken from an arbitrary quadratic polynomial, the subdivision scheme renders this same polynomial. This can easily be derived using the degree elevation algorithm of piecewise polynomials.

The proof uses the following lemma:

Lemma 4.1. Given a subdivision scheme that generates piecewise polynomial functions of degree $d$ at each level. The triangles are subdivided according to a regular PowellSabin split.

Consider one such a triangle at any level, with vertices $x_{0}, x_{1}, x_{2}$ with each $x_{j} \in \mathbb{R}^{2}$. Denote $X=\left\{x_{j}\right\}_{j=0}^{2}$, and $C(X)$ as the convex hull of $X$.

Let $b_{i_{1}, i_{2}}\left(i_{1}, i_{2} \in \mathbb{N}\right.$ with $\left.0 \leqslant i_{1}+i_{2} \leqslant d\right)$ denote the Bézier net of $s \in \pi_{d}$, i.e.,

$$
s(x)=\sum_{0 \leqslant i_{1}+i_{2} \leqslant d} \frac{d !}{\left(d-i_{1}-i_{2}\right) ! i_{1} ! i_{2} !} b_{i_{1}, i_{2}} \prod_{k=0}^{2} \tau_{k}^{i_{k}},
$$

where $\tau_{k}=\operatorname{det}\left(X_{k}\right) / \operatorname{det}(X)$. Here $X_{k}$ denotes the set $X$ with $x_{k}$ replaced by $x$.

Then the norms

$$
\|b\|:=\max _{i_{1}, i_{2}}\left|b_{i_{1}, i_{2}}\right| \text { and }\|s\|_{\infty}:=\sup _{x \in C(X)}|s(x)|
$$

are equivalent:

$$
\|b\| \sim\|s\|_{\infty}
$$

i.e., there exist constants $0<C_{1} \leqslant C_{2}<\infty$, such that

$$
C_{1}\|b\| \leqslant\|s\|_{\infty} \leqslant C_{2}\|b\| \text {. }
$$




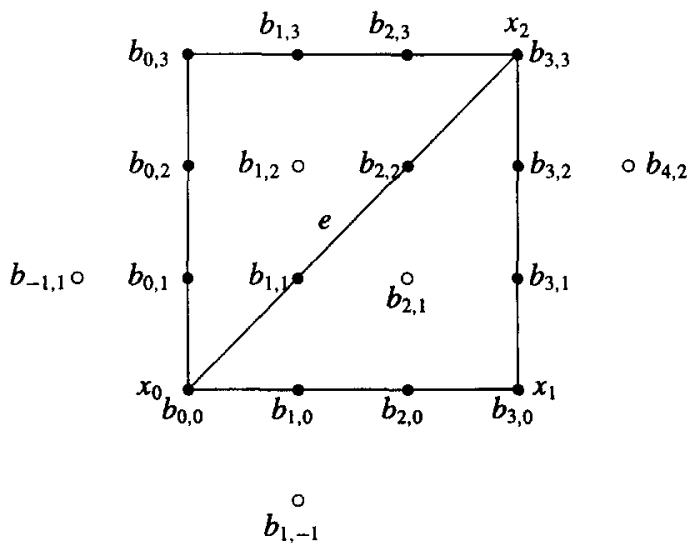

Fig. 11. The Bézier points involved for the refinement of one triangle $\left(x_{0}, x_{1}, x_{2}\right)$.

The constants $C_{i}$ do not depend on the iteration level, but only on the initial triangulation of the data.

Proof. The lemma is trivial for a fixed triangulation. Indeed $s \equiv 0 \Leftrightarrow b_{i_{1}, i_{2}}=0, \forall i_{1}, i_{2}$. A simple scaling argument shows that the coefficients $C_{i}$ can only depend on the angles within the triangles that are present in the triangulation. Due to the fact that a regular Powell-Sabin split in every step is used, these angles are equal at each level and hence the constants $C_{i}$ do not depend on the iteration level.

\subsection{Type I triangulation - continuity proof}

A type I triangulation is a triangulation where the mesh-lines are given by $x=i, y=i$ and $x-y=i, \forall i \in \mathbb{Z}$, and the convergence of the subdivision scheme described in the previous section is examined.

The simplest way to describe the subdivision scheme, is to rewrite it in terms of the Bézier net of an element from the space $S_{3}^{0}$. At each subdivision step a continuous cubic spline is associated with the known function values and both derivatives in each vertex. Then at some level of iteration we have in some specific triangle a Bézier net given (see Fig. 11).

Note that it would be formally more correct to write $b_{k, m}^{i}$ in stead of $b_{k, m}$ if we are considering the $i$ th triangle. However, superfluous indices are omitted in case there can be no confusion.

We further agreed to put (see (2.3)):

$$
\begin{aligned}
b_{2,1}= & \frac{1}{3}\left(\alpha\left(b_{1,1}+b_{1,0}+b_{2,2}+b_{2,0}+b_{3,2}+b_{3,1}\right)\right. \\
& \left.+(1-2 \alpha)\left(b_{0,0}+b_{3,0}+b_{3,3}\right)\right) .
\end{aligned}
$$

A subdivision creates from each triangle four new ones, from which we can distinguish two essential different ones: type $A_{k}, k=1,2,3$, and type $B$, as shown in Fig. 12 . 


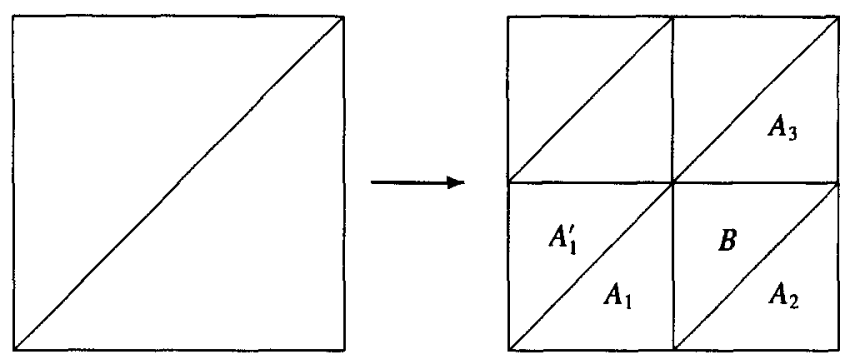

Fig. 12. The Powell-Sabin split in two adjacent triangles.

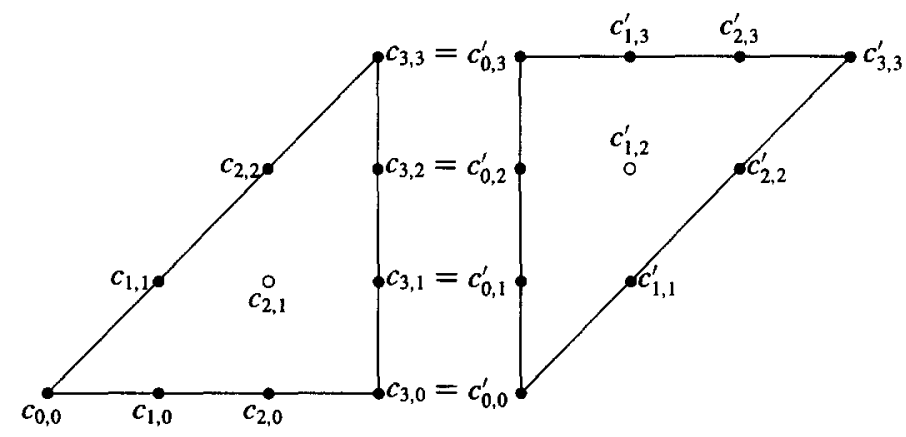

Fig. 13. Bézier net after iteration $n+1$ in a triangle of type $A$ (left) and $B$ (right).

The Bézier points in each subsequent step are linear combinations of the old ones: consider triangle $A_{1}$ with its Bézier points as depicted in the left of Fig. 13. Each $c$ is a linear combination of $b_{i, j}$ with $0 \leqslant j \leqslant i \leqslant 3$ and the three midpoints in the three adjacent triangles $\left(b_{1,-1}, b_{4,2}\right.$ and $\left.b_{1,2}\right)$ as shown in Fig. 11 .

Most of the numbers $c_{i, j}$ are simple to compute, as they follow from the standard subdivision rules (the boundary curves remain intact):

$$
\begin{aligned}
c_{0,0} & =b_{0,0}, \\
c_{1,0} & =\frac{1}{2}\left(b_{0,0}+b_{1,0}\right), \\
c_{2,0} & =\frac{1}{4}\left(b_{0,0}+2 b_{1,0}+b_{2,0}\right), \\
c_{3,0} & =\frac{1}{8}\left(b_{0,0}+3 b_{1,0}+3 b_{2,0}+b_{3,0}\right),
\end{aligned}
$$

and similar expressions for $c_{k, k}, k=1,2,3$. Furthermore, a little algebra shows that averaging of the normal derivatives in the middle of the edges implies

$$
\begin{aligned}
& c_{3,2}=\frac{1}{8}\left(-b_{1,2}+b_{0,0}+b_{1,0}+3 b_{1,1}+b_{2,1}+2 b_{2,2}+b_{3,2}\right), \\
& c_{3,1}=\frac{1}{8}\left(-b_{1,-1}+b_{0,0}+3 b_{1,0}+2 b_{2,0}+b_{1,1}+b_{2,1}+b_{3,1}\right) .
\end{aligned}
$$


Eq. (4.1) then leads to

$$
\begin{aligned}
c_{2,1}= & \frac{1}{24}\left(-\alpha\left(b_{1,-1}+b_{1,2}\right)+b_{0,0}(10-6 \alpha)+\left(b_{1,0}+b_{1,1}\right)(6 \alpha+3)\right. \\
& +\left(b_{2,0}+b_{2,2}\right)(3-2 \alpha)+\left(b_{3,0}+b_{3,3}\right)(1-2 \alpha) \\
& \left.+\alpha\left(2 b_{2,1}+b_{3,1}+b_{3,2}\right)\right), \\
c_{1,2}^{\prime}= & \frac{1}{24}\left(-2\left(b_{1,2}+b_{1,-1}+b_{4,2}\right) \alpha+2(1-\alpha)\left(b_{0,0}+b_{3,0}+b_{3,3}\right)\right. \\
& \left.+(3+\alpha)\left(b_{1,0}+b_{2,0}+b_{1,1}+b_{2,2}+b_{3,2}+b_{3,1}\right)+6 b_{2,1} \alpha\right) .
\end{aligned}
$$

Theorem 4.2. Consider a type I triangulation with given data in the vertices: a function value and both derivatives. Together with (4.1) this fixes a Bézier net $\{b\}$. Then repeatedly applying the subdivision scheme defined by Eqs. (4.2)-(4.4) converges to a $C^{0}$-function for all $\alpha$ satisfying $-1 \leqslant \alpha<2$.

Proof. Consider two subsequent subdivision steps, $n$ and $n+1$. After $n$ steps there are $T_{n}=4^{n} T_{0}$ triangles, and the data on the coarser grid can be represented by a Bézier net $\left\{b^{i}\right\}_{i=1}^{T}$. Similarly Bézier nets $\left\{c^{i}\right\}_{i=1}^{4 T}$ are defined on the finer grid. Both Bézier nets represent splines from $S_{3}^{0}$ and hence the distance can be computed between the spline represented by $\left\{b^{i}\right\}_{i=1}^{T}$ and $s^{n+1}$ given by $\left\{c^{i}\right\}_{i=1}^{4 T}$. First $\left\{b^{i}\right\}_{i=1}^{T}$ is subdivided in the 'regular' way, i.e., the triangle is split into four parts and the four equivalent Bézier nets are computed, giving $\left\{d^{i}\right\}_{i=1}^{4 T}$. The phrase 'equivalent Bézier net' stands for a Bézier net that represents the same spline-function, but on the finer grid.

Applying lemma 4.1 the distance between the $n$th and $(n+1)$ th patch is easily obtained:

$$
\varepsilon_{n}=\left\|s^{n}-s^{n+1}\right\|_{\infty} \leqslant C \sup _{i, j, k}\left|d_{j, k}^{i}-c_{j, k}^{i}\right| \text {. }
$$

For short we write

$$
\Delta_{1} b_{j, k}:=b_{j, k}-b_{j+1, k}, \quad \Delta_{2} b_{j, k}:=b_{j, k}-b_{j, k+1}, \quad \Delta_{3} b_{j, k}:=b_{j, k}-b_{j+1, k+1} .
$$

Without any computation one can show that for all $i, j, k$ the quantity $d_{j, k}^{i}-c_{j, k}^{i}$ can be expressed in terms of differences of nearest neighbours in $\left\{b^{i}\right\}_{i=1}^{T}$,

$$
d_{j, k}^{i}-c_{j, k}^{i}=\sum_{m, n, p, q} \gamma_{m, n, p, q}^{i, j, k} \Delta_{m} b_{p, q}^{n}
$$

for some numbers $\gamma_{m, n, p, q}^{i, j, k}$, from which only a finite number is non-zero, given $i, j, k$. This follows from the locality of the scheme and the fact that any new Bézier point is a convex combination of Bézier points of the previous generation.

If the maximum distance between all neighbouring Bézier points after the $n$th step equals $\delta_{n}$ :

$$
\delta_{n}:=\sup _{i, j, k, m}\left|\Delta_{m} b_{j, k}^{i}\right|,
$$

it suffices to show that

$$
\delta_{n+1}=\sup _{i, j, k, m}\left|\Delta_{m} c_{j, k}^{i}\right| \leqslant \lambda \delta_{n} \quad \text { for some } \lambda<1,
$$


because then

$$
\varepsilon_{n} \leqslant C \sup _{i, j, k}\left|d_{j, k}^{i}-c_{j, k}^{i}\right| \leqslant C^{\prime} \delta_{n} \leqslant C^{\prime \prime} \lambda^{n},
$$

and using standard arguments (as in (Dyn et al., 1987)) this is sufficient for convergence to a $C^{0}$ function.

We now concentrate on one particular triangle in the $n$th step, and superfluous indices are omitted. The numbers $b_{i, j}$ are the old ones (see Fig. 11) and $c_{i, j}, c_{i, j}^{\prime}$ (Fig. 13) denotes the Bézier nets of triangle $A_{1}$, respectively $B$ of the refinement (as defined in Fig. 12). Because of the symmetry, only these have to considered.

So for each nearest neighbour pair in the refined triangulation, it has to be proved that the difference is strictly smaller than the maximum over all differences in the old triangulation. Differences of Bézier points on the 'old' edges necessarily obey this relation, e.g.,

$$
\begin{aligned}
\left|c_{2,2}-c_{3,3}\right| & =\frac{1}{8}\left|b_{0,0}+b_{1,1}-b_{2,2}-b_{3,3}\right| \\
& \leqslant \frac{1}{8}\left(\left|\Delta_{3} b_{0,0}\right|+2\left|\Delta_{3} b_{1,1}\right|+\left|\Delta_{3} b_{2,2}\right|\right) \leqslant \frac{1}{2} \delta_{n} .
\end{aligned}
$$

In fact all differences in two neighbouring Bézier points, not involving the mid-points $c_{2,1}$ or $c_{1,2}^{\prime}$, can similarly be bounded.

There are three essentially different terms with $c_{2,1}: \Delta_{3} c_{1,0}=c_{1,0}-c_{2,1}, \Delta_{2} c_{2,0}=$ $c_{2,0}-c_{2,1}$ and $\Delta_{1} c_{2,1}=c_{2,1}-c_{3,1}$. Finally in the type $B$ triangle there is only one essentially different term left, namely $\Delta_{2} c_{1,1}^{\prime}=c_{1,1}^{\prime}-c_{1,2}^{\prime}$.

The following example of an estimate shows how to get a range for $\alpha$ for which there is convergence to a continuous function. It holds that

$$
\begin{aligned}
24\left|c_{1,0}-c_{2,1}\right|= & \mid b_{1,-1} \alpha+b_{1,2} \alpha+2 b_{0,0}(3 \alpha+1)+3 b_{1,0}(3-2 \alpha)+b_{2,0}(2 \alpha-3) \\
& +b_{3,0}(2 \alpha-1)-3 b_{1,1}(1+2 \alpha)-2 b_{2,1} \alpha-b_{3,1} \alpha+b_{2,2}(2 \alpha-3) \\
& -b_{3,2} \alpha+b_{3,3}(2 \alpha-1) \mid
\end{aligned}
$$

and by expressing the Bézier points outside the triangle in terms of Bézier points and differences thereof inside, i.e.,

$$
b_{1,-1}=b_{1,0}+\Delta_{2} b_{1,-1}, \quad b_{1,2}=b_{1,1}-\Delta_{2} b_{1,1},
$$

gives that

$$
\begin{aligned}
24\left|c_{1,0}-c_{2,1}\right| \leqslant & \mid b_{1,0}(9-5 \alpha)+\left(b_{2,0}+b_{2,2}\right)(2 \alpha-3)+(\alpha-1)\left(b_{3,1}+b_{3,2}+b_{1,1}\right) \\
& -2 b_{2,1} \alpha|+2| \Delta_{3} b_{0,0}(3 \alpha+1)\left|+\left(\left|\Delta_{2} b_{1,-1}\right|+\left|\Delta_{2} b_{1,1}\right|\right)\right| \alpha \mid \\
& +\left(\left|\Delta_{2} b_{3,0}\right|+\left|\Delta_{2} b_{3,2}\right|\right)|2 \alpha-1| .
\end{aligned}
$$

Now a further estimate is made by writing the Bézier points as follows

$$
b_{0,0}=b_{1,1}+\Delta_{3} b_{0,0}, \quad b_{3,0}=b_{3,1}+\Delta_{2} b_{3,0}, \quad b_{3,3}=b_{3,2}-\Delta_{2} b_{3,2},
$$

which yields 


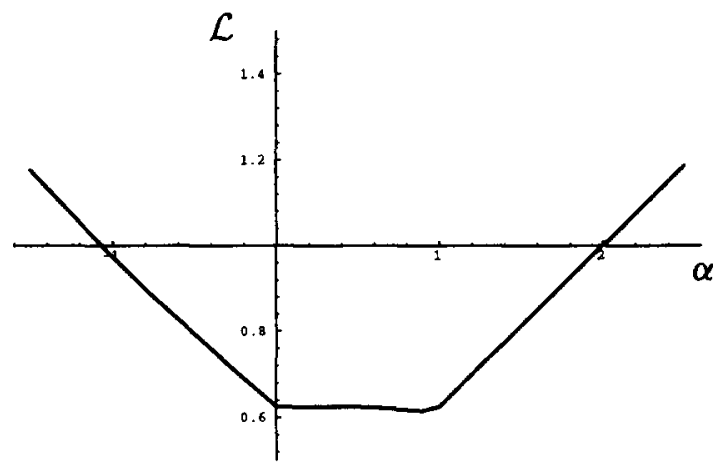

Fig. 14. The optimal $\mathcal{L}$ as defined in (4.7) as a function of $\alpha$.

$$
\begin{aligned}
24\left|c_{1,0}-c_{2,1}\right| \leqslant & (2|3 \alpha+1|+2|\alpha|+2|2 \alpha-1|+|9-5 \alpha|+2|2 \alpha-3| \\
& +3|\alpha-1|) \delta_{n} .
\end{aligned}
$$

Now simple algebra shows that

$$
-\frac{1}{6}<\alpha<\frac{12}{7} \Rightarrow\left|c_{1,0}-c_{2,1}\right|<\delta_{n} \text {. }
$$

Similar estimates pretty straightforwardly give the following range $-1 / 6<\alpha<4 / 3$ for which the subdivision scheme converges to a $C^{0}$ function.

This estimate of the range of $\alpha$ is not optimal. For example in expressing $b_{1,-1}$ in terms of Bézier points and its differences, the following choice was made:

$$
b_{1,-1}=b_{1,0}+\Delta_{2} b_{1,-1} \text {, }
$$

but equally possible is the following identity:

$$
b_{1,-1}=b_{2,0}+\Delta_{3} b_{1,-1} \text {, }
$$

or even better, a linear combination of both right hand sides. The estimate of the range of possible $\alpha$ 's may in this way become better.

In order to improve the bounds numerical software was used, by writing it in terms of a linear programming problem. Consider $\Delta_{i} c_{j, k}$ for the cases above, i.e., $\{i, j, k\}=$ $\{3,1,0\},\{2,2,0\},\{1,2,1\},\{2,4,1\}$ for a given $\alpha$. Then also fix all the differences of Bézier points we want to express $\Delta_{i} c_{j, k}$ in-the larger the number of differences, the better the estimates of the bounds may become. Here only the Bézier points in the triangle at hand were considered, plus its three neighbouring triangles. Next an equality of the following kind is imposed:

$$
\Delta_{i} c_{j, k}=\sum_{p, q, r} w_{p, q, r} \Delta_{p} b_{q, r}+\sum_{m} \lambda_{m} f_{m}(b)+\sum_{m} \mu_{m} c_{m}(b),
$$

where $f_{m}$ and $c_{m}$ are identically zero. For example

$$
\begin{aligned}
f_{1}(b)=F\left(b_{2,1}\right):= & \frac{1}{3}\left(\alpha\left(b_{11}+b_{10}+b_{22}+b_{20}+b_{32}+b_{31}\right)\right. \\
& \left.+(1-2 \alpha)\left(b_{0,0}+b_{3,0}+b_{3,3}\right)\right)-b_{2,1}
\end{aligned}
$$


and similar expressions for $b_{1,2}, b_{4,2}$ and $b_{1,-1}$ respectively. Furthermore there are $10 C^{1}$ conditions on the Bézier points, e.g.,

$$
c_{1}(b)=2 b_{0,0}-b_{0,1}-b_{0,-1} \text {. }
$$

Eq. (4.5) can be seen as a number (28 to be exact) of linear constraints on the coefficients $w, \lambda$ and $\mu$ : both sides are linear expressions in $\{b\}$. Now we look for a minimum of

$$
\mathcal{L}=\sum_{p, q, r}\left|w_{p, q, r}\right|
$$

such that (4.5) is satisfied and $f_{m}=0, m=1, \ldots, 4, c_{m}=0, m=1, \ldots, 10$. Using software to solve linear optimisation problems, one can increase the bounds on $\alpha$ such that the resulting surface is $C^{0}$ (see Fig. 14):

$$
-1 \leqslant \alpha<2 \Rightarrow \mathcal{L}<1 .
$$

\subsection{Type I triangulation-smoothness proof}

Several papers have been written on smoothness investigations of linear subdivision schemes. Indeed, even the butterfly scheme (Dyn et al., 1990), elegant because of its simplicity, is not always differentiable in the initial data-see also (Ball and Storry, 1988; Reif, 1995). The problems are mainly due to the fact that the schemes are parametric and not functional. For example in (Reif, 1995) for certain subdivision schemes local selfintersections are found, and that is (by construction) impossible in the subdivision schemes which are dealt with in this section: investigating functional schemes is in fact much simpler.

In the subdivision scheme which is investigated here, at each level $n$ a piecewise polynomial representation of our function is known, call it $f^{n}$, which is continuous. That implies that the derivatives of this function $f_{x}^{n}$ and $f_{y}^{n}$ are both piecewise discontinuous functions, and these discontinuities occur at edges. First the following convergence theorem is proved, and finally it is shown in Theorem 4.4 that the resulting function $f^{\infty}$ is in fact $C^{1}$.

Theorem 4.3. Consider a type I triangulation with data given in the vertices: a function value and both derivatives. Together with (4.1) this fuxes a Bézier net $\{b\}$. Then repeatedly applying the subdivision scheme defined by equations (4.2-4.4) converges to a $C^{0}$-function, for which also both derivatives converge for all $\alpha$ satisfying $1 / 4 \leqslant \alpha \leqslant 3 / 4$.

Proof. It is sufficient to show that

$$
\varepsilon_{n}^{\prime}=\left\|s_{x}^{n}-s_{x}^{n+1}\right\|_{\infty}+\left\|s_{y}^{n}-s_{y}^{n+1}\right\|_{\infty} \leqslant C \sup _{i, j, k, \ell}\left|\Delta_{\ell} d_{j, k}^{i}-\Delta_{\ell} c_{j, k}^{i}\right|
$$

converges. Similar as in the proof of convergence in the previous subsection, this implies that it is sufficient that the quantities

$$
\delta_{n}^{\prime}:=2^{n} \sup _{i, j, k, \ell, m}\left|\Delta_{\ell} \Delta_{m} b_{j, k}^{i}\right|
$$


satisfy

$$
\delta_{n+1}^{\prime}=2^{n+1} \sup _{j, k, \ell, m}\left|\Delta_{\ell} \Delta_{m} c_{j, k}\right| \leqslant \lambda^{\prime} \delta_{n}^{\prime}, \quad \text { for some } \lambda^{\prime}<1 .
$$

In other words we need to determine the values of $\alpha$ for which one can prove that

$$
\left|\Delta_{i} \Delta_{j} c_{k, \ell}\right|<\frac{1}{2} \sup _{m, n, p, q}\left|\Delta_{m} \Delta_{n} b_{p, q}\right|, \quad \forall i, j, k, \ell .
$$

Again the number of intermediate proofs that is required, in order to achieve this result, is enormous. Therefore we again took our resort to a numerical optimisation program. Like before we put

$$
\Delta_{i} \Delta_{j} c_{k, \ell}=\sum_{m, n, p, q} w_{m, n, p, q} \Delta_{m} \Delta_{n} b_{p, q}+\sum_{m} \lambda_{m} f_{m}(b)+\sum_{m} \mu_{m} c_{m}(b) .
$$

From this the linear constraints and the expressions for the goal functions

$$
\mathcal{L}=\sum_{m, n, p, q}\left|w_{m, n, p, q}\right|
$$

were automatically derived with the symbolic program Reduce (Hearn, 1991). Finally $C^{1}$ continuity was again proved using numerical software for $1 / 4<\alpha \leqslant 3 / 4$.

Having established convergence, it is now proved that it the resulting derivative functions are continuous, in other words that the limiting function $f^{\infty}$ is of class $C^{1}$.

Theorem 4.4. Given a subdivision scheme that uses a regular Powell-Sabin split for the spatial coordinates and that at each level $n$ can be represented by a piecewise continuous polynomial function $f^{n}$, which is $C^{1}$ in all vertices. Suppose the following assumptions hold:

(1) Convergence of function values: The sequence of functions $f^{n}$ satisfy

$$
\left\|f^{n+1}-f^{n}\right\|_{\infty}<C \lambda^{n}
$$

for some $\lambda<1$.

(2) Convergence of both derivative values: The sequence of functions $f_{x}^{n}$ and $f_{y}^{n}$ satisfy

$$
\left\|f_{x}^{n+1}-f_{x}^{n}\right\|_{\infty}+\left\|f_{y}^{n+1}-f_{y}^{n}\right\|_{\infty}<C^{\prime} \lambda^{\prime n}
$$

for some $\lambda^{\prime}<1$.

Then the limiting function $f^{\infty}=\lim _{n \rightarrow \infty} f^{n}$ exists and it is $C^{1}$ smooth.

Proof. Clearly by assumptions (1) and (2), the functions $f^{n}, f_{x}^{n}$ and $f_{y}^{n}$ converge. It remains to show that both $f_{x}^{\infty}$ and $f_{y}^{\infty}$ are continuous. This can be proved using the smoothness in the vertices at each level.

The minimal diameter $h_{n}$ of a triangulation $\mathcal{T}_{n}$ at level $n$ is defined as

$$
h_{n}:=\min _{T \in \mathcal{T}_{n}} \sup \{\operatorname{diam}(S) \mid S \text { is a circle contained in triangle } T\},
$$

and as a regular Powell-Sabin split is used, it holds that $h_{n}=2^{-n} h_{0}$. 


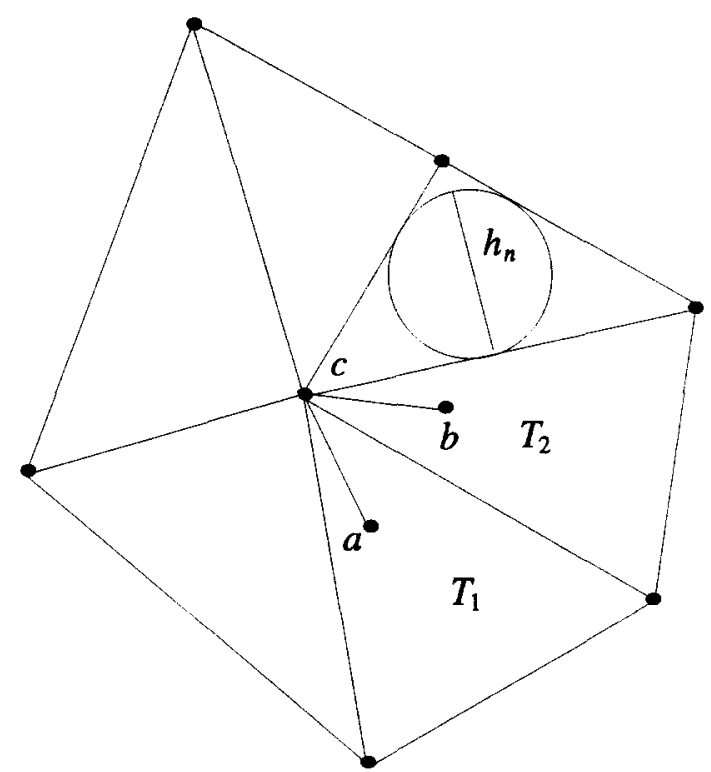

Fig. 15. Continuity proof of the derivatives.

A function $g$ is continuous if for each $\varepsilon>0$ there exists a $\delta>0$ such that

$$
|g(a)-g(b)|<\varepsilon \text { for all }\|a-b\|<\delta .
$$

Now we prove continuity of $f_{x}^{\infty}$ (of course $f_{y}^{\infty}$ can be done similarly). For any $n$ it holds that

$$
\left|f_{x}^{\infty}(a)-f_{x}^{\infty}(b)\right| \leqslant\left|f_{x}^{\infty}(a)-f_{x}^{n}(a)\right|+\left|f_{x}^{n}(a)-f_{x}^{n}(b)\right|+\left|f_{x}^{n}(b)-f_{x}^{\infty}(b)\right| .
$$

Due to assumption 2 the first and the last term can be estimated:

$$
\left|f_{x}^{\infty}(a)-f_{x}^{n}(a)\right|+\left|f_{x}^{n}(b)-f_{x}^{\infty}(b)\right| \leqslant 2 C^{\prime} \lambda^{\prime n} /\left(1-\lambda^{\prime}\right) .
$$

Now take $n$ such that $2 C^{\prime} \lambda^{\prime n} /\left(1-\lambda^{\prime}\right)<\varepsilon / 2$. Furthermore take any $\delta<h_{n}$ and denote $T_{1}, T_{2}$ as the triangles in which $a$, respectively $b$ lie-see Fig. 15. If $T_{1}=T_{2}$ there is nothing to prove as within patches all functions are polynomial, and hence continuous.

As $\delta<h_{n}, T_{1}$ and $T_{2}$ at least share one vertex, located say at position $c \in D$. Then

$$
\left|f_{x}^{n}(a)-f_{x}^{n}(b)\right| \leqslant\left|f_{x}^{n}(a)-f_{x}^{n}(c)\right|+\left|f_{x}^{n}(c)-f_{x}^{n}(b)\right| .
$$

Both terms can be bounded by $\varepsilon / 4$ by taking $\delta$ small enough, as $f_{x}$ is continuous along the path $a-c-b$. In other words it can be achieved that

$$
\left|f_{x}^{\infty}(a)-f_{x}^{\infty}(b)\right| \leqslant \varepsilon / 2+2 \varepsilon / 4=\varepsilon .
$$

\subsection{Proof of convergence for scattered data}

Seeing the result of the previous subsection, it seems an immense task to prove convergence in a more general setting, namely when the data are scattered. Apart from $\alpha$ 


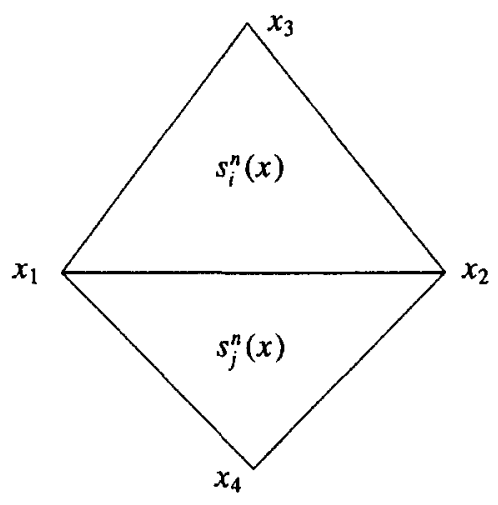

Fig. 16. Two triangles from the scattered data.

we have at least 6 more parameters (the relative position of the three vertices describing the adjacent triangles). However, in the case that $\alpha=3 / 4$ the proof turns out to be quite simple.

As before denote $\{b\}$ as the Bézier net after the $n$th iteration, $\{d\}$ its equivalent on the finer grid, and $\{c\}$ as the Bézier net after the subsequent iteration. Now consider two adjacent triangles with vertices $x_{1}, x_{2}, x_{3}$ and $x_{2}, x_{1}, x_{4}$ respectively as in Fig. 16.

As before

$$
\varepsilon_{n} \leqslant C \sup _{i, j, k}\left|d_{j, k}^{i}-c_{j, k}^{i}\right| \leqslant C \lambda^{n} \quad \text { for some } \lambda<1
$$

guarantees convergence to a continuous function. Furthermore

$$
\varepsilon_{n}^{\prime} \leqslant C \sup _{i, j, k, \ell}\left|\Delta_{\ell} d_{j, k}^{i}-\Delta_{\ell} c_{j, k}^{i}\right| \leqslant C^{\prime} \lambda^{\prime n} \quad \text { for some } \lambda^{\prime}<1
$$

guarantees convergence of the derivatives of the function. The proof that these limiting derivative functions are also continuous (and hence $f^{\infty}$ in $C^{1}$ ) has already been proved in Theorem 4.4.

A suitable quantity to compute everything with is the jump of the normal derivative in the middle of each edge: take $e=\left(x_{1}, x_{2}\right), x_{i} \in \mathbb{R}^{2}$, and put for the jump in the first derivative $\widehat{J}_{e}^{n}:=J_{1,2}$ :

$$
\begin{aligned}
J_{1,2} & :=\left.\frac{2}{3\left\|x_{2}-x_{1}\right\|^{2}} x_{\perp} \cdot D_{x}\left(s_{i}^{n}(x)-s_{j}^{n}(x)\right)\right|_{x=\left(x_{1}+x_{2}\right) / 2} \\
& =\frac{b_{1,0} V_{234}+b_{2,0} V_{143}-b_{2,1} V_{142}-b_{1,-1} V_{123}}{V_{142} V_{123}},
\end{aligned}
$$

with $x_{\perp}=\left(x_{2}^{2}-x_{1}^{2}, x_{1}^{1}-x_{2}^{1}\right)$, where upper index 1,2 stand for the direction 1 respectively 2. Moreover $V_{i j k}$ denotes twice the area of a triangle with vertices $x_{i}, x_{j}, x_{k}$. The odd looking normalisation $\left\|x_{2}-x_{1}\right\|^{-2}$ adapted in this expression is chosen to arrive at a simple expression in terms of only Bézier points and areas of triangles. 
The behaviour of this quantity as a function of the level $n$ turns out to control the convergence of function values as well as derivative values. First the new Bézier net in terms of the old one (in one subdivision step) is computed.

The computation of the new Bézier net $\{c\}$ in terms of the old one $\{b\}$ is straightforward: points on the old wire frame $\left\{c_{k, 0}\right\}_{k=0}^{3}$ are equal to the value to the regular subdivision $\{d\}$, as in (4.2). The Bézier net of the spline equivalent to $\{b\}$ on the refined Bézier net is of course known. Indeed the remaining $d$ 's are given by:

$$
\begin{aligned}
& d_{3,1}=\frac{1}{8}\left(b_{3,1}+2 b_{2,1}+b_{2,0}+b_{1,1}+2 b_{1,0}+b_{0,0}\right), \\
& d_{2,1}=\frac{1}{4}\left(b_{2,1}+b_{1,1}+b_{1,0}+b_{0,0}\right), \\
& d_{1,2}^{\prime}=\frac{1}{8}\left(b_{3,2}+b_{3,1}+b_{2,2}+2 b_{2,1}+b_{2,0}+b_{1,1}+b_{1,0}\right),
\end{aligned}
$$

and all other $d$ 's follow from symmetry. The subdivision scheme generates a sequence of $C^{0}$ cubic splines, which are $C^{1}$ in the vertices (the wireframe is $C^{1}$ ). $C^{1}$ continuity in $\left(x_{1}+x_{2}\right) / 2$ implies that

$$
c_{2,0} V_{234}+c_{3,0} V_{143}-c_{3,1} V_{142}-c_{2,-1} V_{123}=0
$$

Therefore, as one easily checks, averaging of the normals implies that the expression for $c$ for a given $d$ should look as follows:

$$
c_{3,1}=d_{3,1}+\delta V_{123}, \quad c_{2,-1}=d_{2,-1}+\delta V_{142}
$$

and the $C^{\mathbf{l}}$ condition halfway the edge yields a value for $\delta$ :

$$
\delta=\frac{d_{2,0} V_{234}+d_{3,0} V_{143}-d_{3,1} V_{142}-d_{2,-1} V_{123}}{2 V_{142} V_{123}}
$$

If the spline given by the Bézier net $\{b\}$ (and hence also $\{d\}$ ) is smooth along this edge, this correction $\delta$ is zero, i.e., $\delta$ should be proportional to $J_{1,2}$. This is the detailed proof of this:

$$
\begin{aligned}
16 V_{142} V_{123} \delta= & \left(V_{234} b_{0,0}+V_{143} b_{1,0}-V_{142} b_{1,1}-V_{123} b_{0,-1}\right) \\
& +2\left(V_{234} b_{1,0}+V_{143} b_{2,0}-V_{142} b_{2,1}-V_{123} b_{1,-1}\right) \\
& +V_{234} b_{2,0}+V_{143} b_{3,0}-V_{142} b_{3,1}-V_{123} b_{2,-1} \\
& +\left(V_{234}+V_{143}-V_{142}-V_{123}\right)\left(b_{0,0}+2 b_{1,0}+b_{2,0}\right) \\
= & 2 V_{142} V_{123} J_{1,2} \Rightarrow \delta=\frac{1}{8} J_{1,2}
\end{aligned}
$$

as $V_{234}+V_{143}-V_{142}-V_{123}=0$. Hence

$$
c_{3,1}=d_{3,1}+\frac{V_{123}}{8} J_{1,2}
$$


The new midpoints $c_{2,1}$ and $c_{1,2}^{\prime}$ also follow from (4.1) with $\alpha=3 / 4$ :

$$
\begin{aligned}
c_{2,1}= & \frac{1}{4}\left(d_{1,0}+d_{2,0}+d_{1,1}+d_{2,2}+d_{3,1}+d_{3,2}\right)-\frac{1}{6}\left(d_{0,0}+d_{3,0}+d_{3,3}\right) \\
& +\frac{1}{32}\left(J_{1,2}+J_{1,3}\right) V_{123} \\
= & \frac{1}{96}\left(-2 b_{3,3}+3 b_{3,2}+3 b_{3,1}-2 b_{3,0}+3 b_{2,2}+12 b_{2,1}+3 b_{2,0}+27 b_{1,1}\right. \\
& \left.+27 b_{1,0}+22 b_{0,0}\right)+\frac{1}{32}\left(J_{1,2}+J_{1,3}\right) V_{123} \\
= & \frac{1}{4}\left(b_{0,0}+b_{1,0}+b_{1,1}+b_{2,1}\right)+\frac{1}{32}\left(J_{1,2}+J_{1,3}\right) V_{123} \\
= & d_{2,1}+\frac{V_{123}}{32}\left(J_{1,2}+J_{1,3}\right) .
\end{aligned}
$$

From the second to the third line, equation (4.1) with $\alpha=3 / 4$ was used for $b_{2,1}$. Similarly it follows that

$$
c_{1,2}^{\prime}=d_{1,2}^{\prime}+\frac{V_{123}}{16}\left(J_{1,2}+J_{1,3}+J_{2,3}\right)
$$

Remark 4.5. In the case that the triangulation is of type I (as in the previous section) (4.8)-(4.11) reduce to (4.3) and (4.4) (put $V_{j k \ell}=C$ ).

Theorem 4.6. Consider an arbitrary triangulation with data given in the vertices: a function value and both derivatives. Together with (4.1) this fixes a Bézier net $\{b\}$. Then repeatedly applying the subdivision scheme defined by (4.2) and (4.9)-(4.11) converges to a $C^{1}$-function.

Proof. As a regular Powell-Sabin split is used we have that $V_{i j k}=\mathrm{O}\left(4^{-n}\right)$. Therefore from (4.2) and (4.9)-(4.11) it can be inferred that

$$
\sup _{i, j, k}\left|d_{j, k}^{i}-c_{j, k}^{i}\right| \leqslant 4^{-n} C \sup _{e}\left|\widehat{J}_{e}^{n}\right|
$$

and

$$
2^{n} \sup _{i, j, k, \ell}\left|\Delta_{\ell} d_{j, k}^{i}-\Delta_{\ell} c_{j, k}^{i}\right| \leqslant 2^{-n} 2 C \sup _{e}\left|\widehat{J}_{e}^{n}\right|
$$

as, e.g.,

$$
\left|\Delta_{1} d_{j, k}^{i}-\Delta_{1} c_{j, k}^{i}\right| \leqslant\left|d_{j, k}^{i}-c_{j, k}^{i}\right|+\left|d_{j+1, k}^{i}-c_{j+1, k}^{i}\right| .
$$

Here we now investigate the quantity $\sup _{e}\left|J_{e}^{n}\right|$. Only two different cases have to be considered (see Fig. 17): all other cases follow from symmetry. Consider a jump $K_{1}$ of de Bézier net $\{c\}$ over the edge $\left(x_{1}, x_{2}\right)$ :

$$
\begin{aligned}
\frac{V_{142} V_{123}}{4 \cdot 4} K_{1}= & c_{1,0} \frac{V_{234}}{4}+c_{2,0} \frac{V_{143}}{4}-c_{2,1} \frac{V_{142}}{4}-c_{1,-1} \frac{V_{123}}{4} \\
= & \frac{1}{4}\left(d_{1,0} V_{234}+d_{2,0} V_{143}-d_{2,1} V_{142}-d_{1,-1} V_{123}\right. \\
& \left.-\frac{1}{32} V_{123} V_{142}\left(2 J_{1,2}+J_{1,3}+J_{1,4}\right)\right) .
\end{aligned}
$$




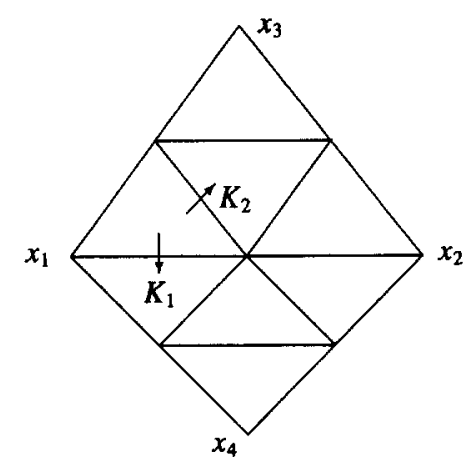

Fig. 17. Jumps in first derivatives.

Here equation (4.8) was used. Now, if we put

$$
\delta:=\frac{d_{1,0} V_{234}+d_{2,0} V_{143}-d_{2,1} V_{142}-d_{1,-1} V_{123}}{V_{142} V_{123}}
$$

some straightforward computation using (4.6) gives

$$
\begin{aligned}
8 V_{142} V_{123} \delta= & 2\left(V_{234} b_{0,0}+V_{143} b_{1,0}-V_{142} b_{1,1}-V_{123} b_{0,-1}\right) \\
& +2\left(V_{234} b_{1,0}+V_{143} b_{2,0}-V_{142} b_{2,1}-V_{123} b_{1,-1}\right) \\
& +2\left(V_{234}+V_{143}-V_{142}-V_{123}\right)\left(b_{0,0}+b_{1,0}\right) \\
& -V_{142} F\left(b_{2,1}\right)-V_{123} F\left(b_{1,-1}\right) \\
= & 2 V_{142} V_{123} J_{1,2} \Rightarrow \delta=\frac{1}{4} J_{1,2}
\end{aligned}
$$

and so

$$
K_{1}=\frac{1}{8}\left(6 J_{1,2}-J_{1,3}-J_{1,4}\right) .
$$

Similarly for the other case, it was found that (the triangles are congruent in this case):

$$
K_{2}=\frac{c_{3,1}+c_{3,2}-c_{2,1}-c_{1,2}^{\prime}}{\frac{1}{4} V_{123}},
$$

or, as $\{d\}$ is $C^{1}$ within one 'old' triangle, it is straightforward to verify that

$$
K_{2}=\frac{1}{8}\left(J_{1,2}+J_{1,3}-2 J_{2,3}\right) \text {. }
$$

Combining (4.14) and (4.15), it is now proved that

$$
\sup _{e}\left|\widehat{J}_{e}^{n+1}\right|=\max \left(\left|K_{1}\right|,\left|K_{2}\right|\right) \leqslant \sup _{e}\left|\widehat{J}_{e}^{n}\right| \text {. }
$$

This estimate, together with (4.12) and (4.13) proves convergence of the limiting function and both its derivatives. The proof that the derivatives converge to a continuous function is essentially the same as in the previous section, Theorem 4.4 . 
Remark 4.7. If one starts with data drawn from a spline $s \in S_{3}^{1}$, the subdivision scheme with $\alpha=3 / 4$ will return a spline $s^{\prime} \in S_{3}^{1}$. It is not necessarily true that $s=s^{\prime}$. After the first step however, all Bézier nets will be equivalent. (A choice that does reproduce the same element at all levels will be discussed in the final section: this method is less attractive as it is less local.)

Due to this phenomenon the desired estimates for the distance of Bézier nets in two subsequent subdivision levels can therefore be expressed in the jumps of the first derivatives perpendicular to the edges. If one can give sufficiently strong estimates on these jumps (as was done here), convergence of function values, convergence of the derivatives, and continuity of these functions is immediate (see Theorem 4.4). Essentially this theorem shows that functional schemes are much easier to investigate than parametric schemes.

Remark 4.8. In Theorem 4.4 it seems essential that the method gives a sequence of splines that are $C^{1}$ in the vertices. This is not true, however. Having established strong enough bounds on the jumps of the derivatives over the edges, Theorem 4.4 can also be proved by examining the straight path $a-b$ in place of $a-c-b$ (see Figure 15).

\section{Higher order schemes}

A natural question emerges from the previous section: is there an extension to schemes which render even smoother surfaces? It turns out to be possible to derive a $C^{2}$ subdivision scheme based on the spline space $S_{5}^{1}$, which admits a local basis. In contrast with the cubic method in the previous section however, the regularity of the triangulation turns out to be important: it should not be 'too irregular'.

Given a triangulation, at each vertex function values and first and second order derivatives are assumed to be given, as well as the normal first derivative halfway each edge (again, if not, one can of course estimate them). This fixes $3 \times 6$ Bézier points from the data at the vertices, plus 3 from the normal derivatives halfway the edge, and it fixes uniquely an element from the space $S_{5}^{1}$.

A step in the subdivision scheme is now to take a Powell-Sabin split, taking all the necessary function values and first derivatives from the patch, and the second order derivatives from an averaging of the two adjacent patches (if necessary):

1. The function values, first and second order derivatives in the new vertices are equal to the average of these values in the old step, at that position.

2. The normal first derivative halfway the new edge is the average of the two values in the old step, also at that position.

Note that in contrast with the cubic method described in the previous section, there is no free parameter left. A consequence is that if one draws data from an element $s \in S_{5}^{2}$, the scheme reproduces this same element with this subdivision scheme, and therefore, analogous to before, the behaviour of the second derivative across edges is investigated. For $e=\left(x_{1}, x_{2}\right)$ we define

$$
\widehat{J}_{e}^{n}(x):=\frac{1}{2\left\|x_{2}-x_{1}\right\|^{4}}\left(x_{\perp} \cdot D_{x}\right)^{2}\left(s_{i}^{n}(x)-s_{j}^{n}(x)\right) .
$$


As for each $n$ the spline is $C^{1}$ across the edges and $C^{2}$ in the vertices, the discontinuity in the second derivative on this edge can be characterised by the following two quantities:

$$
\begin{aligned}
\widehat{J}_{e, 1}^{n}= & \frac{1}{V_{142}^{2} V_{123}^{2}}\left(b_{3,2} V_{142}^{2}-2 b_{3,1} V_{142} V_{143}+b_{3,0} V_{143}^{2}\right. \\
& \left.-2 b_{2,1} V_{142} V_{234}+2 b_{2,0} V_{143} V_{234}+b_{1,0} V_{234}^{2}-b_{1,-2} V_{123}^{2}\right), \\
\widehat{J}_{e, 2}^{n}= & \frac{1}{V_{142}^{2} V_{123}^{2}}\left(b_{4,2} V_{142}^{2}-2 b_{4,1} V_{142} V_{143}+b_{4,0} V_{143}^{2}\right. \\
& \left.-2 b_{3,1} V_{142} V_{234}+2 b_{3,0} V_{143} V_{234}+b_{2,0} V_{234}^{2}-b_{2,-2} V_{123}^{2}\right),
\end{aligned}
$$

where

$$
\begin{aligned}
& \widehat{J}_{e, 1}^{n}=\frac{2}{3} \widehat{J}_{e}^{n}\left(\frac{2 x_{1}+x_{2}}{3}\right)+\frac{1}{3} \widehat{J}_{e}^{n}\left(\frac{x_{1}+2 x_{2}}{3}\right), \\
& \widehat{J}_{e, 2}^{n}=\frac{1}{3} \widehat{J}_{e}^{n}\left(\frac{2 x_{1}+x_{2}}{3}\right)+\frac{2}{3} \widehat{J}_{e}^{n}\left(\frac{x_{1}+2 x_{2}}{3}\right) .
\end{aligned}
$$

Indeed, one can easily show that

$$
\sup _{0 \leqslant \lambda \leqslant 1}\left|\widehat{J}_{e}^{n}\left(\lambda x_{1}+(1-\lambda) x_{2}\right)\right| \sim\left|\widehat{J}_{e, 1}^{n}\right|+\left|\widehat{J}_{e, 2}^{n}\right| \text {. }
$$

Now

$$
\varepsilon_{n+1}^{\prime \prime}:=4^{-n-1} \sup _{e, i}\left|\widehat{J}_{e, i}^{n+1}\right| \leqslant \lambda^{\prime \prime} \varepsilon_{n}^{\prime \prime} \quad \text { for some } \lambda^{\prime \prime}<1
$$

guarantees a $C^{2}$-surface. Indeed similarly as in the previous section one can prove that the method reproduces elements from the space $S_{5}^{2}$ and hence the distance between Bézier nets of two subsequent levels can all be expressed in terms of the jumps in the second derivatives across edges. Then convergence of functions and first and second derivatives and continuity of these limiting functions are then easily proved, using an extension of Theorem 4.4 (see Remark 4.7).

In contrast with the algorithm in the previous section, it turns out that the regularity of the grid comes into play. Consider Fig. 18 and put

$$
\xi_{k \ell m}:=\frac{\sin \phi_{k} \cos \phi_{\ell}}{\sin \phi_{m}}, \quad \widehat{\xi}_{k \ell m}:=\frac{\sin \psi_{k} \cos \psi_{\ell}}{\sin \psi_{m}}, \quad \forall\{k, \ell, m\}=\{1,2,3\} .
$$

The result found with the aid of Reduce (Hearn, 1991), was that (see figure 18 for definitions):

$$
\begin{aligned}
K_{1}= & -2 J_{1}, \\
K_{2}= & \frac{1}{4}\left(-\left(\xi_{321}+\widehat{\xi}_{321}+2\right) J_{1}+\left(2-\xi_{321}-\widehat{\xi}_{321}\right) J_{2}\right. \\
& \left.+\left(2+\xi_{321}\right)\left(J_{5}+J_{6}\right)-\left(2+\widehat{\xi}_{321}\right)\left(J_{7}+J_{8}\right)\right), \\
K_{3}= & \frac{1}{4}\left(\left(2 \xi_{321}-\xi_{312}\right)\left(J_{1}+J_{2}\right)-\xi_{132}\left(J_{3}+J_{4}\right)-\left(1+2 \xi_{321}\right)\left(J_{5}+J_{6}\right)\right),
\end{aligned}
$$

and the expression for $K_{4}$ as well as all other jumps follow from symmetry. 


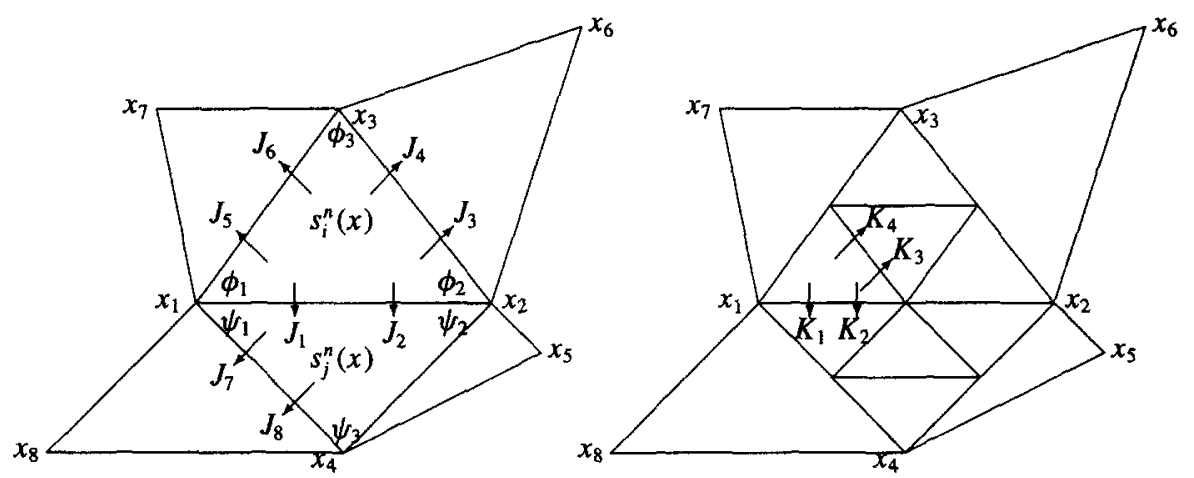

Fig. 18. Jumps in second derivatives.

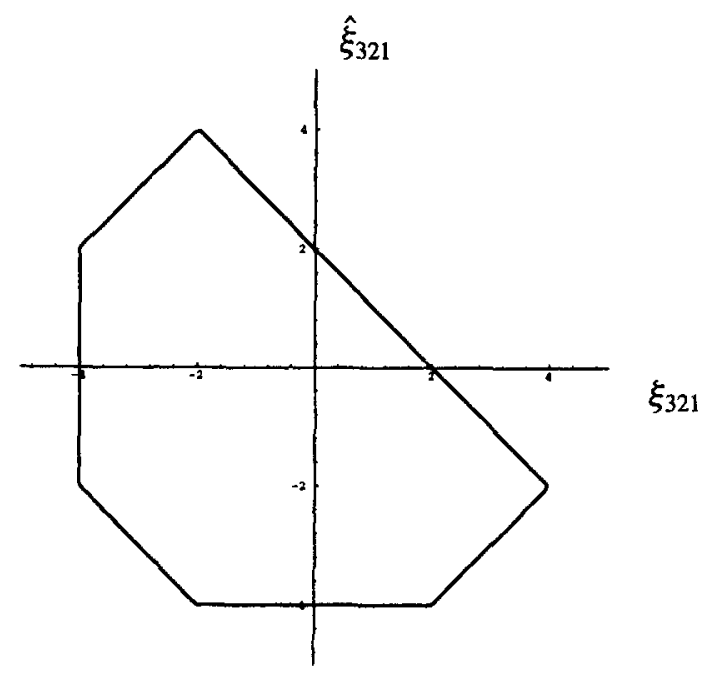

Fig. 19. Values $\left(\xi_{321}, \widehat{\xi}_{321}\right)$ for which $\left|K_{2}\right|<4 \sup _{e}\left|J_{e}\right|$.

It should hold that $\left|K_{\ell}\right|<4 \sup _{e}\left|J_{e}\right|, \ell=1, \ldots, 3$, as given in (5.2). Fig. 19 shows the regions in $\xi_{321}, \widehat{\xi}_{321}$-space for which $K_{2}$ satisfies this requirement: the boundary of the region is given by:

$$
\left|\xi_{321}+\widehat{\xi}_{321}+2\right|+\left|2-\xi_{321}-\widehat{\xi}_{321}\right|+2\left|2+\xi_{321}\right|+2\left|2+\widehat{\xi}_{321}\right|=16 .
$$

From this expression it is simple to prove that it is sufficient to demand that

$$
-3<\xi_{k \ell m}, \widehat{\xi}_{k \ell m}<1, \quad \forall k, \ell, m .
$$

By restricting this a little further, i.e.,

$$
-\frac{4}{3}<\xi_{k \ell m}, \widehat{\xi}_{k \ell m}<1, \quad \forall k, \ell, m
$$


also $K_{3}$ satisfies the same restriction:

$$
\left|K_{3}\right|<\frac{1}{4}\left(2 \cdot \frac{11}{3}+2 \cdot \frac{4}{3}+2 \cdot 3\right) \sup _{e}\left|J_{e}\right|=4 \sup _{e}\left|J_{e}\right| .
$$

In other words the regularity condition (5.3) is sufficient for $C^{2}$ convergence.

Note that even for the simplest triangulations the result need not be $C^{2}$ : for a type I triangulation it holds that $\xi_{k \ell m}=1$ for some $k, \ell, m$. Moreover the method is not affine invariant: only transformations which conserve angles yield the resulting surface invariant. This is due to the choice of the normal derivative halfway the edge as an average of the normals of the two patches. Alternatively we could have chosen an averaging of a directional derivative: a choice that is affine invariant (cf., Fig. 18) is to set

$$
\begin{aligned}
\left(x_{3}-x_{4}\right) \cdot D_{x} s^{n+1}\left(\frac{3 x_{1}+x_{2}}{4}\right)= & \frac{1}{2}\left(x_{3}-x_{4}\right) \cdot D_{x}\left(s_{i}^{n}\left(\frac{3 x_{1}+x_{2}}{4}\right)\right. \\
& \left.+s_{i}^{n}\left(\frac{3 x_{1}+x_{2}}{4}\right)\right),
\end{aligned}
$$

and similarly for all other edges. The conditions for $C^{2}$ change in the following way. Again the method maps the space $S_{5}^{2}$ on to itself, and the formulas for the jumps in the second derivatives become:

$$
\begin{aligned}
K_{1}= & -2 J_{1}, \\
K_{2}= & \frac{1}{4}\left(-\left(2+\rho_{1}+\rho_{1}^{\prime}\right) J_{1}+\left(2-\rho_{1}-\rho_{1}^{\prime}\right) J_{2}+\left(2+\rho_{1}\right)\left(J_{5}+J_{6}\right)\right. \\
& \left.-\left(2+\rho_{1}^{\prime}\right)\left(J_{7}+J_{8}\right)\right), \\
K_{3}= & \frac{1}{4}\left(\left(2 \rho_{1}-\rho_{2}\right)\left(J_{1}+J_{2}\right)+\rho_{3}\left(J_{3}+J_{4}\right)-\left(1+2 \rho_{1}\right)\left(J_{5}+J_{6}\right)\right) .
\end{aligned}
$$

Here

$$
\rho_{1}=\frac{V_{126}}{V_{123}+V_{263}}, \quad \rho_{2}=\frac{V_{142}+V_{123}}{V_{143}}, \quad \rho_{3}=\frac{V_{234}}{V_{143}}, \quad \rho_{1}^{\prime}=\frac{V_{152}}{V_{142}+V_{245}} .
$$

Observe the strong similarity with (5.2). Therefore by demanding $-3<\rho_{1}, \rho_{1}^{\prime}<1$ it is true that $\left|K_{2}\right|<4 \sup _{e}\left|J_{e}\right|$. By symmetry however, this should also hold for $1-\rho_{1}, 1-\rho_{1}^{\prime}$ and therefore

$$
\beta \leqslant \rho_{1}, \rho_{1}^{\prime} \leqslant 1-\beta
$$

for some $0<\beta \leqslant 1 / 2$. If this holds, then from the definition for the other coefficients $\rho_{2}, \rho_{3}$ it is also true that

$$
\frac{1}{1-\beta} \leqslant \rho_{2} \leqslant \frac{1}{\beta}, \quad \frac{\beta}{1-\beta} \leqslant \rho_{3} \leqslant \frac{1-\beta}{\beta},
$$

which gives

$$
\begin{aligned}
& \left|K_{3}\right|<\frac{1}{4}\left(2\left|\frac{1}{\beta}-2 \beta\right|+2\left|\frac{1-\beta}{\beta}\right|+2|1+2(1-\beta)|\right) \underset{e}{\sup _{e}\left|J_{e}\right|} \\
& \left|K_{3}\right|<\frac{1}{4}\left(2\left|2(1-\beta)-\frac{1}{1-\beta}\right|+2\left|\frac{1-\beta}{\beta}\right|+2|1+2(1-\beta)|\right) \sup _{e}\left|J_{e}\right| .
\end{aligned}
$$


Demanding that $\left|K_{3}\right|<4 \sup _{e}\left|J_{e}\right|$ gives possible values for $\beta$ :

$$
\frac{\sqrt{17}-3}{4} \approx 0.280776<\beta \leqslant \frac{1}{2} \text {. }
$$

For regular grids, like type $\mathrm{I}, C^{2}$ smoothness is now guaranteed, but the restriction on $\beta$ is still very restrictive for scattered data sets.

Remark. Possibly by allowing irregular splits, i.e., letting the positions of the new vertices depend on the shapes of the triangles the subdivision could give convergence to a $C^{2}$ function for more irregular data, but this was not investigated any further.

\section{Approximation properties}

In this section the approximation properties of the proposed schemes are discussed.

Definition 6.1. Given a fixed initial triangulation $\mathcal{T}$ and a smooth function $f$ from which the data in the nodes of $\mathcal{T}$ are taken in the following way:

$$
f_{i}=f\left(h x_{i}, h y_{i}\right), \quad f_{x, i}=h f_{x}\left(h x_{i}, h y_{i}\right), \quad f_{y, i}=h f_{y}\left(h x_{i}, h y_{i}\right),
$$

and similarly for higher order derivatives. If the limiting function $s^{\infty}$ satisfies

$$
E_{h}:=\left\|s^{\infty}(x, y)-f(h x, h y)\right\|_{\infty} \leqslant C(\mathcal{T}, f) \cdot h^{p}
$$

provided $f$ is smooth enough, then the method is said to be of order $p$.

For the cubic case, it turns out that with the choice of the mid-point as in (2.3) the approximation order is equal to 3 . Numerically this was found to be true for a range of the parameter $\alpha$ (see Table 1)-here it is only proved for the value $\alpha=3 / 4$.

It is fairly simple to give an estimate for the distance between $s^{0}$ and $f$. Without loss of generality consider a triangle with vertices $(0,0),(0,1)$ and $(1,0)$. Then it follows straightforwardly that

$$
\begin{aligned}
s^{0}(x, y)-f(h x, h y)= & \frac{h^{3}}{12} y x(x-1+y)\left(-3 f_{x y y}-3 f_{x x y}+2 f_{x x x}+2 f_{y y y}\right) \\
& +\mathrm{O}\left(h^{4}\right) \Rightarrow\left\|s^{0}(x, y)-f(h x, h y)\right\|_{\infty} \leqslant C \cdot h^{3} .
\end{aligned}
$$

Here the term $f_{x x x}$ stands for the third derivative of $f$ with respect to $x$ in some point within the triangle, etc. It is clear that along edges the approximation is better than 3 as there Hermite interpolation is employed, which explains the factor $y x(x-1+y)$.

One can prove the same for all first derivatives, e.g.,

$$
\begin{aligned}
s_{x}^{0}(x, y)-h f_{x}(h x, h y)= & \frac{h^{3}}{12} y(2 x+y-1) \\
& \times\left(-3 f_{x y y}-3 f_{x x y}+2 f_{x x x}+2 f_{y y y}\right)+\mathrm{O}\left(h^{4}\right), \\
s_{y}^{0}(x, y)-h f_{y}(h x, h y)= & \frac{h^{3}}{12} x(x-1+2 y) \\
& \times\left(-3 f_{x y y}-3 f_{x x y}+2 f_{x x x}+2 f_{y y y}\right)+\mathrm{O}\left(h^{4}\right) .
\end{aligned}
$$


Table 1

Errors for the test-function $f(x, y)=x^{2}+x y^{2}+y^{4}$ for $\alpha=0$ and $\alpha=3 / 4$.

\begin{tabular}{ccl}
\hline$h$ & $E_{h}(\alpha=0)$ & $E_{h}\left(\alpha=\frac{3}{4}\right)$ \\
\hline 1.000000 & $1.56 \cdot 10^{-1}$ & $1.09 \cdot 10^{-1}$ \\
0.500000 & $9.15 \cdot 10^{-3}$ & $5.86 \cdot 10^{-3}$ \\
0.250000 & $5.37 \cdot 10^{-4}$ & $2.49 \cdot 10^{-4}$ \\
0.125000 & $5.12 \cdot 10^{-5}$ & $3.06 \cdot 10^{-5}$ \\
0.062500 & $5.47 \cdot 10^{-6}$ & $3.98 \cdot 10^{-6}$ \\
0.031250 & $6.32 \cdot 10^{-7}$ & $5.23 \cdot 10^{-7}$ \\
0.015625 & $7.61 \cdot 10^{-8}$ & $6.77 \cdot 10^{-8}$ \\
\hline
\end{tabular}

Together with Eqs. (4.8)-(4.10) it therefore holds that

$$
\left\|s^{\infty}(x, y)-s^{0}(x, y)\right\|_{\infty} \leqslant C^{\prime \prime} \cdot h^{3} .
$$

Indeed

$$
\left\|s^{n+1}(x, y)-s^{n}(x, y)\right\|_{\infty} \leqslant C \sup _{i, j, k}\left|d_{j, k}-c_{j, k}\right| \leqslant C^{\prime} V_{n} \sup _{e}\left|\widehat{J}_{e}^{n}\right| .
$$

Using the estimate in (4.16) this gives

$$
\begin{aligned}
\left\|s^{\infty}(x, y)-s^{0}(x, y)\right\|_{\infty} & \leqslant C^{\prime} \sum_{n=0}^{\infty} V_{n} \sup _{e}\left|\widehat{J}_{e}^{n}\right| \leqslant C^{\prime} \sup _{e}\left|\widehat{J}_{e}^{0}\right| \sum_{n=0}^{\infty} V_{n} \\
& =C^{\prime \prime} \sup _{e}\left|\widehat{J}_{\epsilon}^{0}\right| .
\end{aligned}
$$

From (6.1) we can give an estimate on $\sup _{e}\left|\widehat{J}_{e}^{0}\right|$. Consider an edge $e$ with $s_{i}^{0}, s_{j}^{0}$ as in Fig. 16. Then

$$
\begin{aligned}
\left\|s_{i, x}^{0}(x, y)-s_{j, x}^{0}(x, y)\right\|_{\infty} \leqslant & \left\|s_{i, x}^{0}(x, y)-f(h x, h y)\right\|_{\infty} \\
& +\left\|s_{j, x}^{0}(x, y)-f(h x, h y)\right\|_{\infty} \leqslant C^{\prime} \cdot h^{3},
\end{aligned}
$$

and similarly for the $y$-derivative: hence $\sup _{e}\left|\widehat{J}_{e}^{0}\right| \leqslant C^{\prime \prime} \cdot h^{3}$. Finally

$$
\begin{aligned}
\left\|s^{\infty}(x, y)-f(h x, h y)\right\|_{\infty} \leqslant & \left\|s^{0}(x, y)-f(h x, h y)\right\|_{\infty} \\
& +\left\|s^{\infty}(x, y)-s^{0}(x, y)\right\|_{\infty} \leqslant C^{\prime \prime \prime} \cdot h^{3} .
\end{aligned}
$$

By taking a less local choice for the mid-point, this approximation order can be improved to 4 . One such a choice is to fix the mid-point in the following way: compute for each triangle estimates of the normal derivatives in the middle of the three edges of that triangle, using the values of the three nodal values of the three adjacent nodes. Call the three estimated values $u_{1}, u_{2}$ and $u_{3}$. Then fix $m^{0}$ such that these normal derivatives on average are correct: from the subdivision scheme (and hence from the value of $m^{0}$ ) 
we can formally compute $\widehat{u}_{1}\left(m^{0}\right), \widehat{u}_{2}\left(m^{0}\right)$ and $\widehat{u}_{3}\left(m^{0}\right)$. Then $m^{0}$ follows from the linear equation

$$
u_{1}+u_{2}+u_{3}=\widehat{u}_{1}\left(m^{0}\right)+\widehat{u}_{2}\left(m^{0}\right)+\widehat{u}_{3}\left(m^{0}\right) .
$$

It can be proved that this method reproduces elements from $S_{3}^{1}$ and also that it has approximation order 4.

Finally we conclude with the remark that the quintic method has optimal approximation power, i.e.,

$$
\left\|s^{\infty}(x, y)-f(h x, h y)\right\|_{\infty} \leqslant C h^{6} .
$$

The proof is essentially the same as the one for the cubic case.

\section{Acknowledgement}

The author wishes to thank the K.N.A.W. (Royal Dutch Academy for Science) for their financial support for a visit to Dalian University of Technology (China), where most of present work was performed. He also wishes to thank for the hospitality of Prof. R-H. Wang from this university. Furthermore he is very grateful to both referees for their constructive criticism.

\section{References}

Ball, A.A. and Storry, D.J.T. (1988), Conditions for tangent plane continuity over recursively generated B-spline surfaces, ACM Trans. on Graphics 7, 83-102.

Catmull, E. and Clark, J. (1978), Recursively generated B-spline surfaces on arbitrary topological meshes, Computer-Aided Design 10, 350-355.

Cavaretta, A.S., Dahmen, W. and Micchelli, C.A. (1991), Stationary subdivision, Memoires of the AMS 93, 1-186.

Cavaretta, A.S. and Micchelli, C.A. (1989), The design of curves and surfaces by subdivision algorithms, in: Lyche, T. and Schumaker, L.L., eds., Mathematical Methods in Computer Aided Geometric Design, Academic Press, Boston, 115-153.

Cottin, C. and van Damme, R. (1994), 3D reconstruction of closed objects by piecewise cubic triangular Bézier patches, in: Bowyer, A., ed., Computer-Aided Surface Geometry and Design: The Mathematics of Surfaces IV, Clarendon Press, Oxford, 395-410.

de Boor, C. (1987), B-splines without divided differences, in: Farin, G.E., ed., Geometric Modeling, SIAM, Philadelphia, 21-27.

Dyn, N., Gregory, J.A. and Levin, D. (1987), A 4-point interpolatory subdivision scheme for curve design, Computer Aided Geometric Design 4, 257-268.

Dyn, N., Gregory, J.A. and Levin, D. (1990), A butterfly subdivision scheme for surface interpolation with tension control, ACM Trans. on Graphics 9, 160-169.

Dyn, N., and Levin, D. (1995), Analysis of Hermite-type subdivision schemes, in: Chui, C.K. and Schumaker, L.L., eds., Approximation Theory VIII, Vol. 2: Wavelets and Multilevel Approximation, World Scientific, Singapore, 117-124.

Dyn, N. Levin, D. and Liu, D. (1992), Interpolatory convexity preserving subdivision schemes for curves and surfaces, Computer-Aided Design 24, 211-216. 
Doo, D. and Sabin, M. (1978), Behaviour of recursive division surfaces near extraordinary points, Computer-Aided Design 10, 356-360.

Franke, R. (1982), Scattered data interpolation: tests of some methods, Math. Comp. 38, 181-200. Hearn, A.C. (1991), REDUCE User's Manual 3.4, Rand.

Merrien, J.-L. (1994), Dyadic Hermite interpolation on a triangulation, in: Mason, J.C. and Cox, M.G., eds., Numerical Algorithms 7, J.C.Baltzer AG, 391-410.

Reif, U. (1995), A unified approach to subdivision algorithms near extraordinary vertices, Computer Aided Geometric Design 12, 153-174. 